\title{
Molecular mechanisms of apoptosis and cell selectivity of zinc dithiocarbamates functionalized with hydroxyethyl substituents
}

\begin{abstract}
In the solid state each of three binuclear zinc dithiocarbamates bearing hydroxyethyl groups, $\{\mathrm{Zn}[\mathrm{S} 2 \mathrm{CN}(\mathrm{R}) \mathrm{CH} 2 \mathrm{CH} 2 \mathrm{OH}] 2\} 2$ for $\mathrm{R}=\mathrm{iPr}(1), \mathrm{CH} 2 \mathrm{CH} 2 \mathrm{OH}(2)$, and $\mathrm{Me}(3)$, and an all alkyl species, [Zn(S2CNEt2)2]2 (4), features a centrosymmetric $\{\mathrm{ZnSCS}\} 2$ core with a step topology; both 1 and 3 were isolated as monohydrates. All compounds were broadly cytotoxic, specifically against human cancer cell lines compared with normal cells, with greater potency than cisplatin. Notably, some selectivity were indicated with 2 being the most potent against human ovarian carcinoma cells (cisA2780), and 4 being more cytotoxic toward multidrug resistant human breast carcinoma cells (MCF-7R), human colon adenocarcinoma cells (HT29), and human lung adenocarcinoma epithelial cells (A549). Based on human apoptosis PCRarray analysis, caspase activities, DNA fragmentation, cell apoptotic assays, intracellular reactive oxygen species (ROS) measurements and human topoisomerase I inhibition, induction of apoptosis in HT-29 cells is demonstrated via both extrinsic and intrinsic pathways. Compounds 2-4 activate the p53 gene while 1 activates both p53 and p73. Cell cycle arrest at the $\mathrm{S}$ and G2/M phases correlates with inhibition of HT-29 cell growth. Cell invasion is also inhibited by 1-4 which is correlated with down-regulation of NF- $\kappa B$.
\end{abstract}

Keyword: Zinc; Dithiocarbamate; Cell selectivity; Apoptosis; Cell cycle; Topoisomerase I 